\title{
Serum Magnesium: A Forgotten Electrolyte after Total Thyroidectomy
}

\author{
Adi S Muhammed ${ }^{a}$, Shahrun N Suhaimi ${ }^{a}$, Lee Qi Zheng ${ }^{a}$ \\ ${ }^{a}$ Department of Surgery, UKM Medical Centre, Hospital Canselor Tuanku Muhriz, Malaysia
}

Keywords

hypomagnesaemia, hypoparathyroidism, hypocalcaemia, thyroidectomy

\section{Corresponding Author}

Assoc. Prof. Dr. Shahrun Niza Abdullah

Suhaimi

Department of Surgery, UKM Medical

Centre, Jalan Yaacob Latiff, 56000 Cheras,

Kuala Lumpur, Malaysia.

Tel No: +603-9145 555

E-mail: shahrun72.sn@gmail.com

Received: 16 October 2020; Accepted: 15 December 2020

Doi: https://doi.org/10.31436/imjm.v20i3

\begin{abstract}
Hypocalcemia is a common complication after total thyroidectomy; however, hypomagnesemia is also frequently detected, and its role is often disregarded. We report a 72-year-old woman who underwent total thyroidectomy with neck dissection and subsequently developed atrial fibrillation due to hypocalcemia and hypomagnesemia. The pathophysiology of hypomagnesemia after total thyroidectomy and its significance in calcium homeostasis is described.
\end{abstract}

\section{INTRODUCTION}

Total thyroidectomy is commonly performed for benign and malignant thyroid conditions. A well-known complication of the surgery is hypocalcemia due to injury to the parathyroid glands (PG). The effect can be transient or permanent depending on the severity of the injury. The incidence has been reported to be approximately $50 \%$ for transient hypocalcemia and $2 \%$ for permanent hypocalcemia. ${ }^{1}$ Other than calcium, hypomagnesemia is also observed in post-thyroidectomy patients. The incidence varies greatly from $10 \%$ to $72 \%$ according to reports. ${ }^{2}$ It is often overlooked by clinicians as complex mechanism regulates its homeostasis.

\section{CASE REPORT}

A 72-year-old woman presented with anterior neck swelling for six months associated with hoarseness and choking upon swallowing liquids. Her symptoms had worsened until she developed stridor and was unable to lie flat. Two years prior, she was diagnosed with hyperthyroidism and treated with carbimazole and propranolol.

On examination, her hoarseness and occasional stridor were noticeable. There was a large, hard, and fixed lobulated right thyroid mass measuring $6 \mathrm{~cm} \mathrm{x} 7 \mathrm{~cm}$ causing tracheal deviation to the left. No cervical lymph nodes were palpable. Clinically she was euthyroid.
Flexible laryngoscopy revealed immobile bilateral vocal cords in the paramedian position. Imaging with ultrasound and computed tomography (CT) scan showed a highly suspicious enlarged right thyroid lesion displacing the trachea. There were also multiple lung nodules and enlarged mediastinal nodes suggestive of metastasis (Figure 1). Fine needle aspiration and cytology of the mass indicated a follicular lesion of undetermined significance (FLUS).

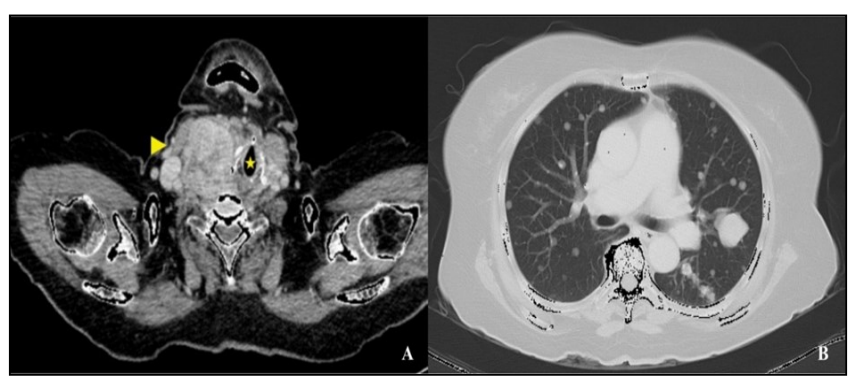

Figure 1: (A) Computed tomography of axial view of the neck showing right thyroid mass (u) and tracheal displacement and narrowing (ó). (B) Axial view of the chest showing mediastinal and multiple lung metastases.

Subsequently, an emergency total thyroidectomy with central neck dissection and tracheostomy was performed. The neck dissection was done as few enlarged central group nodes were found intraoperatively. The tumor was densely adhered to the strap muscles and had invaded the trachea. With regards to the PG, only the left inferior gland was able to be identified and preserved. 
Prior to surgery, results of her blood investigations showed controlled thyroid function and normal calcium and magnesium levels (TSH <0.01 (0.35 - 4.94) UIU/ mL; T4 7.66 (9 - 19.05) pmol/L; Corrected calcium (calcium) 2.45 (2.14 - 2.58) mmol/L; Magnesium $(\mathrm{Mg})$ 0.75 (0.66 - 1.07) mmol/L). Her calcium level was closely monitored after the operation. At 16 hours postop, it dropped slightly to $2.11(2.14-2.58) \mathrm{mmol} / \mathrm{L})$. She was asymptomatic and oral calcium carbonate and calcitriol were commenced.

However, she developed fast atrial fibrillation (AF) on the following day. Her calcium level was lower at 2.01 $(2.14-2.58) \mathrm{mmol} / \mathrm{L}$. She was given intravenous (IV) digoxin for the AF. Another episode of AF occurred on day three post-op and the calcium level was found to have significantly dropped to $1.85(2.14-2.58) \mathrm{mmol} /$ L. Magnesium and potassium levels were also checked and found to be low $(\mathrm{Mg} 0.61(0.66-1.07) \mathrm{mmol} / \mathrm{L}$; $\left.\mathrm{K}^{+} 2.9(3.5-5.1) \mathrm{mmol} / \mathrm{L}\right)$. These were corrected with an IV calcium gluconate, magnesium sulphate, and potassium chloride. She was finally discharged ten days after surgery with normal electrolytes: CCA, 2.43 (2.142.58) $\mathrm{mmol} / \mathrm{L} ; \mathrm{Mg}, 0.81(0.66-1.07) \mathrm{mmol} / \mathrm{L}$; and $\mathrm{K}^{+}$ $4.3(3.5-5.1) \mathrm{mmol} / \mathrm{L}$.

Histopathology of the thyroid specimen revealed a widely invasive follicular thyroid carcinoma with minimal components of poorly differentiated thyroid carcinoma $(\sim 10 \%)$. One node was positive for metastasis and there was tumor infiltration into the trachea. She was consequently referred for radioactive iodine treatment (RAI) and radiotherapy to the neck.

\section{DISCUSSION}

Hypomagnesemia can manifest clinically as cardiovascular or neuromuscular abnormalities such as AF, supraventricular tachycardia, muscle spasms, seizures, and neurocognitive abnormalities such as depression and psychosis. It is commonly observed with other electrolyte abnormalities, such as hypocalcemia and hypokalemia. In post-thyroidectomy patients, this may occur via several pathways.

Parathyroid hormone (PTH) and magnesium have interesting interaction. PTH increases magnesium absorption in the gut and its reabsorption in the renal IMJM Volume 20 No.3, July 2021 tubules. It also facilitates the release of magnesium from the bones where it is stored, into the blood. ${ }^{3}$ Overall, this increases the magnesium concentration in the circulation.

During thyroidectomy especially for malignancy with nodal involvement and local invasion such as this case, the PGs may be accidentally removed during dissection. In the hypoparathyroid state, there will be reduced magnesium absorption in the intestines and reabsorption in the kidneys. Magnesium release from the bones will also be impaired. These responses will result in a net reduction in the circulating magnesium.

A decrease in magnesium may further inhibit PTH production. It may antagonize the effect of calcium in the chief cells. ${ }^{1}$ This was seen in patients with chronic kidney disease who has low magnesium level. Their PTH levels were not as highly elevated as other patients with renal hyperparathyroidism. ${ }^{2}$ In addition, hypomagnesaemia may lead to impaired PTH receptor sensitivity in end-organs making it less potent. ${ }^{4}$ Furthermore, magnesium acts as a cofactor for calcium absorption within the small bowel and participates in vitamin D synthesis. ${ }^{2}$ These effects may exacerbate hypocalcaemia experienced by patients after thyroidectomy.

The interactions among PTH, magnesium, and calcium are depicted in Figure 2. Hypomagnesemia and hypocalcemia have a close correlation and therefore, are often concurrently observed. In a study by Chincholikar, $30 \%$ of post-thyroidectomy patients developed both hypocalcemia and hypomagnesemia. ${ }^{1} \mathrm{It}$ was also noted that patients with both electrolyte abnormalities were more likely to show clinical manifestations. ${ }^{4}$

Interestingly, magnesium is also postulated to exert negative feedback on PTH production and secretion. ${ }^{2}$ It can mimic the effects of calcium at the calcium sensing receptor (CaSR) in parathyroid chief cells. ${ }^{1}$ For example, when magnesium concentration is low, the PG will respond by increasing the production or release of PTH in order to maintain its normal level. However, its affinity is approximately three times lower than that of calcium. ${ }^{2}$ 


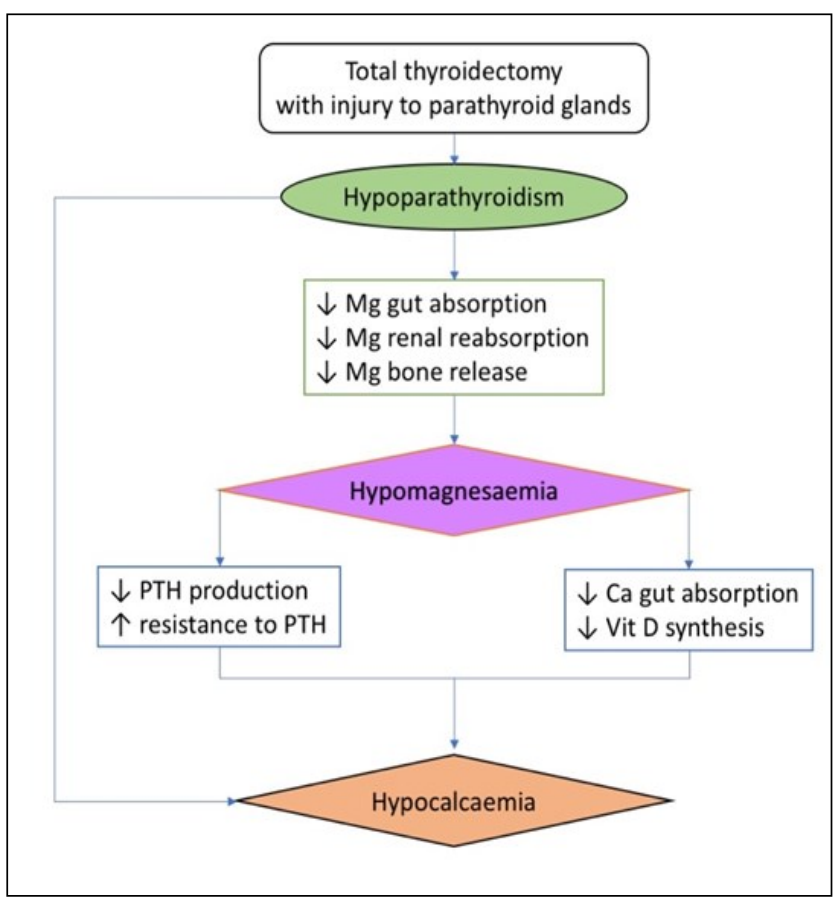

Figure 2: Schematic representation of interactions between thyroidectomy, hypoparathyroidism, hypocalcaemia and hypomagnesaemia.

Patients with hyperthyroidism have been reported to have lower magnesium levels, probably due to increased excretion of magnesium in the urine. ${ }^{4}$ Therefore, it is essential to monitor the electrolytes in patients with toxic multinodular goiter or Graves' disease before and after total thyroidectomy, even though their hyperthyroidism should have been controlled preoperatively.

In a condition called chronic latent magnesium deficiency (CLMD), patients have significantly reduced magnesium storage in the bones due to chronic magnesium deficiency, but they have normal circulating levels of magnesium and are asymptomatic. ${ }^{2}$ They may be underdiagnosed, and physiological stress during surgery may tilt the balance and induce symptomatic hypomagnesemia. Another factor that may contribute to hypomagnesemia is extracellular volume expansion due to perioperative administration of intravenous fluid. This leads to hemodilution and increased renal excretion. $^{2}$

The clinical manifestations of hypomagnesemia and hypocalcemia may overlap. Hypomagnesemia may worsen the symptoms of hypocalcemia and cause correction of hypocalcemia more difficult. When patients develop complications such as AF, longer hospitalization and higher treatment costs are implicated. $^{2}$
Some studies suggest that hypomagnesemia can be used to predict permanent hypocalcemia in postthyroidectomy patients. 4,5 Therefore, clinicians should be alerted to check magnesium level if patients with hypocalcemia respond poorly to treatment. It is also important to recognize patients who are at a higher risk of developing hypocalcemia and hypomagnesemia. Patients going for total thyroidectomy and neck dissection and patients with toxic multinodular goiter or Graves' disease should have their calcium and magnesium levels checked regularly in the postoperative period. However, in a retrospective study by Wang et al, thyroid cancer patients who underwent total thyroidectomy and central neck dissection, hypomagnesemia was not significantly associated with the development of hypocalcemia. ${ }^{6}$ Nonetheless, patients who are prone to develop complications from electrolyte imbalances, such as those with cardiac arrhythmias or epilepsy, should be identified early.

\section{CONCLUSION}

In conclusion, hypomagnesemia should be recognized as a potential complication of total thyroidectomy. Its complex interaction with PTH and calcium metabolism may complicate hypocalcemia that is more commonly observed. It has important clinical significance that may affect surgical outcomes and indirectly increases burden of disease and health costs. Both electrolyte imbalances are easily correctable, hence early detection is essential for prompt treatment and prevention of further complications.

\section{REFERENCES}

1. Chincholikar SP, Ambiger S. Association of Hypomagnesemia with Hypocalcemia after Thyroidectomy. Indian J Endocrinol Metab. 2018 Oct; 22(5):656-60.

2. Meng C, Xie J, Wang W, Xia F, Li X. Hypomagnesemia and post-thyroidectomy hypocalcemia: molecular mechanisms and clinical potential. Int J Clin Exp Med. 2018 Nov; 11 (11):11554-66.

3. Hansen B-A, Bruserud Ø. Hypomagnesemia in critically ill patients. J Intensive Care [Internet]. 2018 Mar 27 [cited 2020 Apr 29];6. Available from: 
https://www.ncbi.nlm.nih.gov/pmc/articles/

PMC5872533/

4. Cherian AJ, Gowri M, Ramakant P, Paul TV, Abraham DT, Paul MJ. The Role of Magnesium in Post-thyroidectomy Hypocalcemia. World J Surg. 2016 Apr;40(4):881-8.

5. Luo H, Yang H, Zhao W, Wei T, Su A, Wang B, et al. Hypomagnesemia predicts postoperative biochemical hypocalcemia after thyroidectomy. BMC Surg. 2017 May;17(1):62.

6. Wang X, Zhu J, Liu F, Gong Y, Li Z.

Postoperative hypomagnesaemia is not associated with hypocalcemia in thyroid cancer patients undergoing total thyroidectomy plus central compartment neck dissection. Int J Surg Lond Engl. 2017 Mar;39:192-6. 